\title{
Rhythmic CLOCK-BMAL1 binding to multiple E-box motifs drives circadian $D b p$ transcription and chromatin transitions
}

\author{
Jürgen A Ripperger ${ }^{1,2}$ \& Ueli Schibler ${ }^{1}$
}

\begin{abstract}
Mammalian circadian rhythms are based on transcriptional and post-translational feedback loops. Essentially, the activity of the transcription factors BMAL1 (also known as MOP3) and CLOCK is rhythmically counterbalanced by Period (PER) and Cryptochrome (CRY) proteins to govern time of day-dependent gene expression ${ }^{1}$. Here we show that circadian regulation of the mouse albumin $D$ element-binding protein (Dbp) gene involves rhythmic binding of BMAL1 and CLOCK and marked daily chromatin transitions. Thus, the Dbp transcription cycle is paralleled by binding of BMAL1 and CLOCK to multiple extra- and intragenic $E$ boxes, acetylation of Lys9 of histone H3, trimethylation of Lys4 of histone $\mathrm{H3}$ and a reduction of histone density. In contrast, the antiphasic daily repression cycle is accompanied by dimethylation of Lys9 of histone $\mathrm{H} 3$, the binding of heterochromatin protein $1 \alpha$ and an increase in histone density. The rhythmic conversion of transcriptionally permissive chromatin to facultative heterochromatin relies on the presence of functional BMAL1-CLOCK binding sites.
\end{abstract}

Circadian rhythms are based on cell-autonomous oscillators that maintain gene expression with a period length of about a day even in the absence of external timing cues ${ }^{2,3}$. In mammals, the rhythmgenerating molecular circuitry is thought to rely on the opposing effects of transcriptional activators and repressors that generate a negative feedback loop ${ }^{1-3}$. Briefly, the PAS domain helix-loop-helix proteins BMAL1 and CLOCK stimulate transcription of Cry and Per via binding to E-box motifs present in these genes. Once the repressor proteins CRY and PER reach a critical concentration, they form complexes with the CLOCK-BMAL heterodimer and thereby attenuate the transactivation potential of these transcription factors. As a consequence, Per and Cry transcription is reduced, PER and CRY accumulation decreases below the concentration required for autorepression and a new transcription cycle of PER and CRY genes can ensue.

The transcription factor DBP shows high-amplitude circadian oscillations ${ }^{4,5}$, and we found that $D b p$ transcription reaches its maximum and minimum at Zeitgeber time (ZT) 7 and ZT19, respectively (Fig. 1a), where ZT0 and ZT12 are the times when lights are switched on and off, respectively. Putative circadian regulatory regions containing E-box motifs have previously been mapped in the $D b p$ locus through the identification of DNase I-hypersensitive sites $^{6}$, and genetic experiments have suggested that at least some of these might serve as binding sites for BMAL1 and CLOCK ${ }^{6,7}$. To examine whether these sites indeed bind CLOCK and BMAL1 in vivo, we have used a chromatin immunoprecipitation (ChIP) technique that we developed for the analysis of solid tissues (see Methods). For simplicity, the Dbp gene was subdivided into nine $\sim 800$-bp segments according to the previously established map of DNase I hypersensitivity sites. ChIP experiments, performed with liver chromatin at 2-h intervals around the clock, suggested that both CLOCK and BMAL1 bind to E-box elements within three regions in a highly circadian fashion (Fig. 1b). The two E-box-containing intronic regions ( 1 and 2) were maximally occupied between ZT6 and ZT8, whereas binding at the promoter element showed a broader temporal distribution, with high occupancy between ZT2 and ZT8. Conceivably, this may reflect a higher affinity of the promoter E-box element for CLOCK and BMAL1 compared with the affinity of the E-box sites within intronic regions, allowing the promoter element to be filled with CLOCK and BMAL1 during a more extended time span.

In accordance with previously published results ${ }^{8}$, our protein blot experiments showed that the accumulation of BMAL1 and CLOCK in mouse liver nuclei made only shallow daily oscillations (Fig. 2a). Hence, the accumulation of these two transcription factors alone could not account for the robust occupancy cycles demonstrated by our ChIP experiments (Fig. 1b). Similarly, when examined in electromobility shift assays (EMSA) under low-stringency conditions (see Methods), liver nuclear proteins formed similar amounts of CLOCKBMAL1-DNA complexes with the promoter E-box motif, irrespective of when the animals had been sacrificed (Fig. 2b). However, EMSA experiments performed under more stringent conditions (see Methods) demonstrated highly circadian binding of CLOCK and BMAL1 to

${ }^{1}$ Department of Molecular Biology and National Center of Competence in Research 'Frontiers in Genetics', Sciences III, University of Geneva, 30, Quai ErnestAnsermet, CH-1211 Geneva 4, Switzerland. ${ }^{2}$ Present address: Section of Biochemistry, Department of Medicine, University of Fribourg, Switzerland. Correspondence should be addressed to U.S. (Ueli.Schibler@molbio.unige.ch).

doi:10.1038/ng1738 


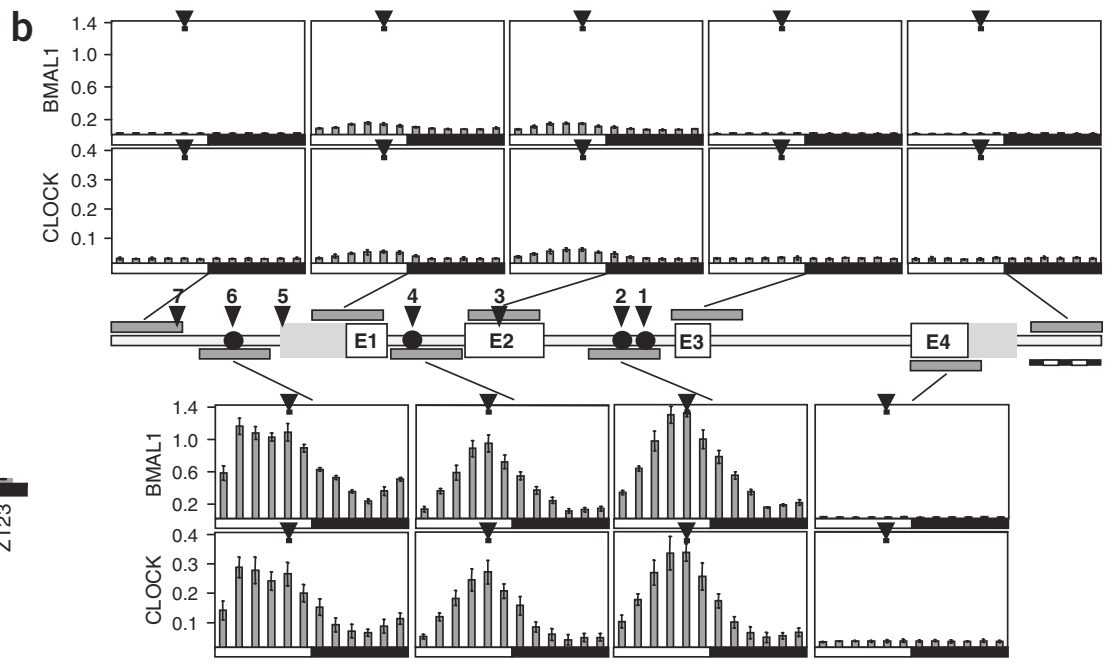

Figure 1 Mapping of binding regions for BMAL1 and CLOCK. (a) Dbp pre-mRNA accumulation in mouse liver was monitored by real-time RT-PCR using primers and a Taqman probe within intron 1. Unspliced pre-mRNAs are rapidly converted to mRNA, and the phase of pre-mRNA thus closely matches the phase of Dbp transcription. Open and filled bars along the $x$-axis symbolize the 12-h light and dark phases, respectively. (b) ChIP analysis for BMAL1 and CLOCK in nine regions of the Dbp gene. ChIP assays were performed with liver chromatin harvested from mice sacrificed at 2-h intervals around the clock. The percentage of immunoprecipitated DNA fragments with regard to an input control are given on the $y$-axis. Shown are averages of four pairwise comparisons ( \pm s.d.). E1 to E4: exons 1 to 4 . Arrowheads numbered 1 to 7: positions of previously mapped DNase I-hypersensitive regions. Arrowheads at the top of graphs mark the time points of highest $D b p$ transcription (ZT7). The black-and-white bar at the far right represents 500 bp.

the same E-box element (Fig. 2c). Therefore, CLOCK and/or BMAL1 must have different DNA binding properties at different times during the day, conceivably owing to different post-translational modifications (see legend to Fig. 2c and ref. 8). The circadian binding of CLOCK and BMAL1 to Dbp E-box elements in vivo and in vitro does not conform to the proposal that these factors remain bound to DNA throughout the day and serve as a chromatin platform for the repressors CRY and PER ${ }^{8,9}$. We favor a scenario in which $\mathrm{CRY}$ and/ or PER either sequester CLOCK-BMAL1 heterodimers into inactive complexes or facilitate post-translational modifications (see ref. 8) that affect the DNA binding of these transcription factors. A similar scenario has been demonstrated recently for the circadian clock of the filamentous fungus Neurospora crassa, in which FRQ (Frequency) attenuates the DNA-binding of WCC (White Collar Complex) by promoting WCC hyperphosphorylation ${ }^{10}$.

Figure 2 Circadian in vitro binding of BMAL1 and CLOCK to the promoter E-box motif. (a) Protein blot analysis. Proteins obtained from mouse liver nuclei at indicated times were visualized with antibodies indicated at left. Filled and open sections of bar at top symbolize the light and dark phases, respectively, and an arrow indicates the peak of $D b p$ transcription. PIP 160 is a nucleolar protein with constant abundance in liver nuclei that served as a loading control. (b) EMSA analysis under low-stringency conditions (see Methods). In the lower panel, an antibody specific for CLOCK was added to the binding reaction to identify complexes containing CLOCK (present in upper panel, absent in lower panel). $\varnothing$ indicates lane without nuclear extract. * indicates specific complexes of unknown composition. B/C: complex containing BMAL1 and CLOCK. U1/2: complex containing Upstream stimulatory factor 1 and 2 (data not shown). (c) EMSA analysis under high-stringency conditions (see Methods). In the lower panel, an antibody specific for BMAL1 was added to the binding reaction to identify complexes containing BMAL1 (present in upper panel, absent in lower panel). Note that the phase of circadian DNA binding in vitro correlates well with that demonstrated by ChIP in Figure $\mathbf{1 b}$ and that of slightly faster migrating CLOCK form in a.
We wished to determine whether the cyclic binding of CLOCK and BMAL1 to cis-acting Dbp regulatory elements elicits daily changes in chromatin structure. To this end, we used ChIP experiments to examine for the presence of signatures diagnostic for transcriptionally permissive chromatin, such as acetylation of Lys9 (H3-K9) and trimethylation of Lys4 (H3-K4) within histone $\mathrm{H} 3$, and for signatures diagnostic for transcriptionally inert chromatin, such as H3-K9 dimethylation and binding of heterochromatin protein 1 ( $\mathrm{Hp} 1$; for review, see refs 11,12). Similar to previous findings on Per1 and Per2 (ref. 9), acetylation of H3-K9 was found to oscillate at the Dbp locus (Fig. 3a), reaching a peak value at times when $D b p$ transcription was maximal. In addition, trimethylation of $\mathrm{H} 3-\mathrm{K} 4$ followed the acetylation pattern of $\mathrm{H} 3-\mathrm{K} 9$, with four- to fivefold amplitude and a slightly delayed peak between ZT8 and ZT10 (Fig. 3a). The spatial distribution of trimethylated $\mathrm{H} 3-\mathrm{K} 4$ at the $\mathrm{Dbp}$ gene is in keeping with the

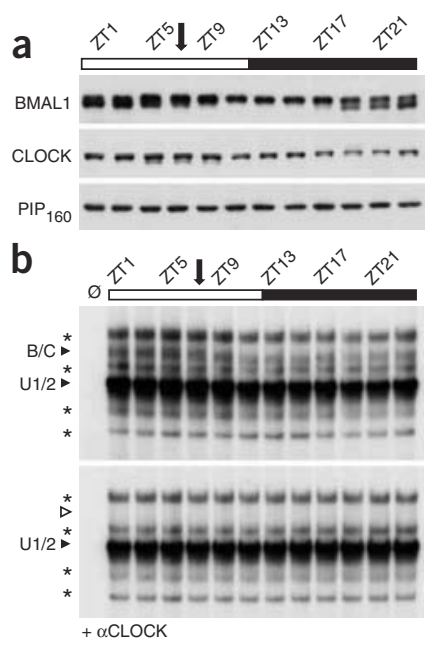

C

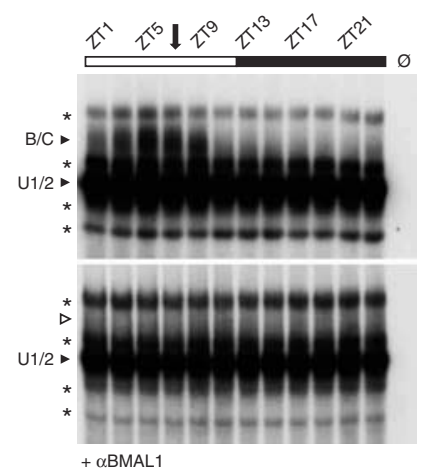




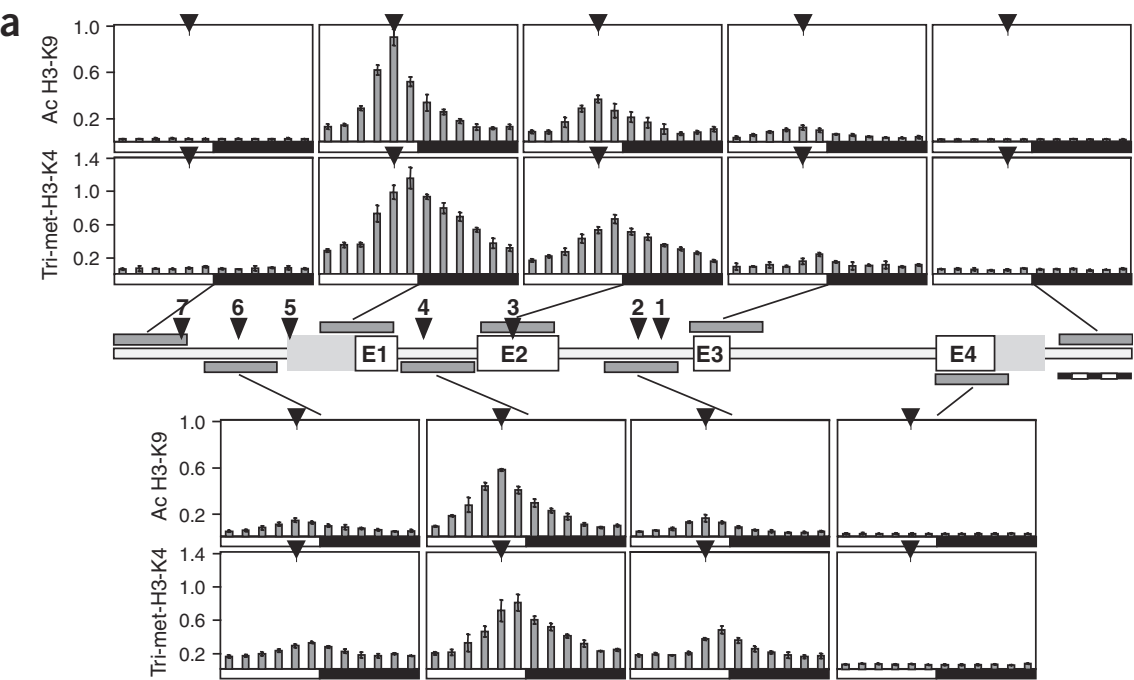

b

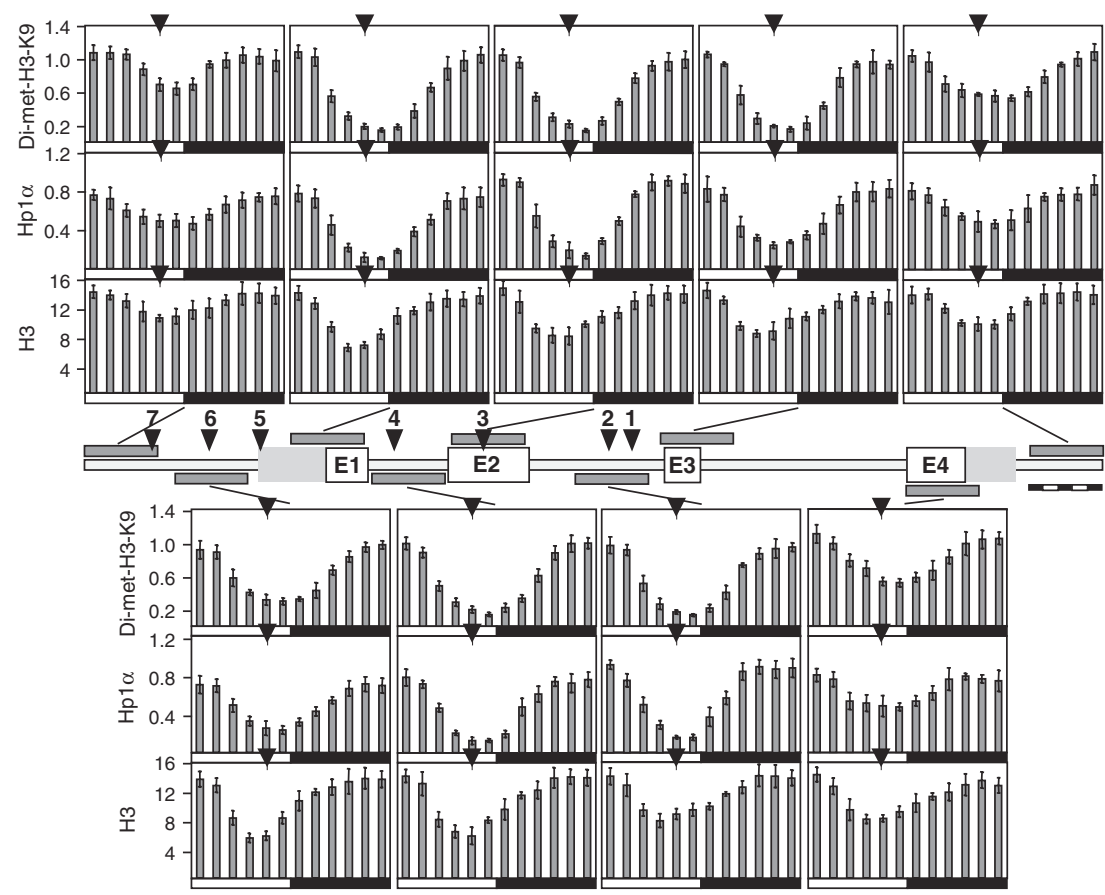

Figure 3 Mapping of circadian histone $\mathrm{H} 3$ modifications and determination of histone $\mathrm{H} 3$ abundance. (a) ChIP using antibodies against acetylated (Ac) H3-K9 and trimethylated (Tri-met) H3-K4. (b) ChIP using antibodies against dimethylated $\mathrm{H} 3-\mathrm{K} 9, \mathrm{Hp} 1 \alpha$ and histone $\mathrm{H} 3$. The data are presented as in Figure $1 \mathrm{~b}$.

proposal that Set1, the responsible methyltransferase, travels with the elongating RNA polymerase II complex some distance along a gene $^{13,14}$. Dimethylation of H3-K9, which is thought to mediate repression of transcription ${ }^{15,16}$, was detected in antiphase to the acetylation of the same residue (Fig. $3 \mathbf{b}$ ). In addition, alternate cycles in H3-K9 acetylation and dimethylation were dependent on a functional molecular oscillator, as they were not observed in arrhythmic Per1 ${ }^{-1-}$ Per $2^{\mathrm{Brdm} / \mathrm{Brdm}}$ mice (Supplementary Fig. 1 online). However, the spatial distribution of these two antiphasic chromatin modifications differed. Circadian H3-K9 acetylation started around the transcriptional initiation site and then gradually decreased until it reached background levels around exon 3. In contrast, the levels of dimethy- lated H3-K9 flanking the $\mathrm{Dbp}$ gene fluctuated with low daily amplitude, and marked circadian changes were observed only at regions where alternate acetylation occurred. The presence of high amounts of dimethylated H3-K9 at the borders of the $\mathrm{Dbp}$ gene correlated with the presence of methylated $\mathrm{CpG}$ sites in this region (Supplementary Fig. 2 online), in keeping with the finding that $\mathrm{H} 3-\mathrm{K} 9$ methylation and DNA methylation can be coupled ${ }^{17}$.

Dimethylation of $\mathrm{H} 3-\mathrm{K} 9$ provides specific binding sites for heterochromatin proteins (Hp1), and binding of $\mathrm{Hp} 1$ to modified histones may engender the subsequent compaction of the chromatin fiber ${ }^{15,16}$. This would offer a plausible explanation for the augmented resistance to DNase I digestion observed within the $D b p$ locus during repression $^{6}$. Hence, we wished to analyze the binding of $\mathrm{Hpl} \alpha$ to the $\mathrm{Dbp}$ locus (Fig. 3b). Indeed, the occupancy of $\mathrm{Hp} 1 \alpha$ closely followed the presence of dimethylated H3-K9 along the $D b p$ gene. On the basis of these observations, it is tempting to speculate that the $D b p$ locus becomes compacted to a facultative heterochromatin state every day. This scenario was further supported by a ChIP analysis with an antibody against histone $\mathrm{H} 3$ (Fig. 3b). These experiments suggested that the density of histone $\mathrm{H} 3$, and supposedly that of nucleosomes in general, fluctuated with approximately twofold amplitude within the gene and the proximal promoter region but showed only minor differences further upstream and downstream of the $D b p$ locus.

Circadian transcription cycles can be recorded in synchronized fibroblast cell lines such as NIH3T3 and Rat-1 (refs. 18-20). To examine the influence of the E-box sequences on $D b p$ expression, we used FLP-mediated site-specific recombination to generate two stable Rat-1 fibroblast cell lines, Rat-1$D b p^{\mathrm{WT}}$ and Rat-1-Dbp $p^{\Delta \mathrm{E}}$, which contain a single $D b p$ transgene integrated in the same orientation and at the same chromosomal locus $^{21}$. Rat-1-Dbp ${ }^{\mathrm{WT}}$ bears a 7-kb genomic DNA fragment spanning the mouse Dbp wildtype allele and all previously identified DNase I-hypersensitive regions associated with it ${ }^{6}$. Rat-1-Dbp ${ }^{\Delta \mathrm{E}}$ contains an equivalent stretch of genomic DNA sequence in which the four E-box motifs have been inactivated by site-specific mutagenesis (E-boxes in promoter and intron 1) or a short deletion (two E-boxes in intron 2). In synchronized Rat-1-Dbp ${ }^{\mathrm{WT}}$ cells, the circadian accumulation of the transgene-derived RNA closely followed that of endogenous Dbp transcripts (Fig. 4a). Therefore, we concluded that the 7-kb fragments contained all regulatory information necessary for circadian expression of the $D b p$ transgene in Rat-1 fibroblasts. Circadian transcription is still observed in a Rat-1 cell line expressing a transgene without the high-affinity promoter E-box (Supplementary Fig. 3 online). However, the transgene of Rat-1-Dbp $p^{\Delta \mathrm{E}}$ cells, which lacks all four E-boxes, is transcribed at very low levels throughout the 


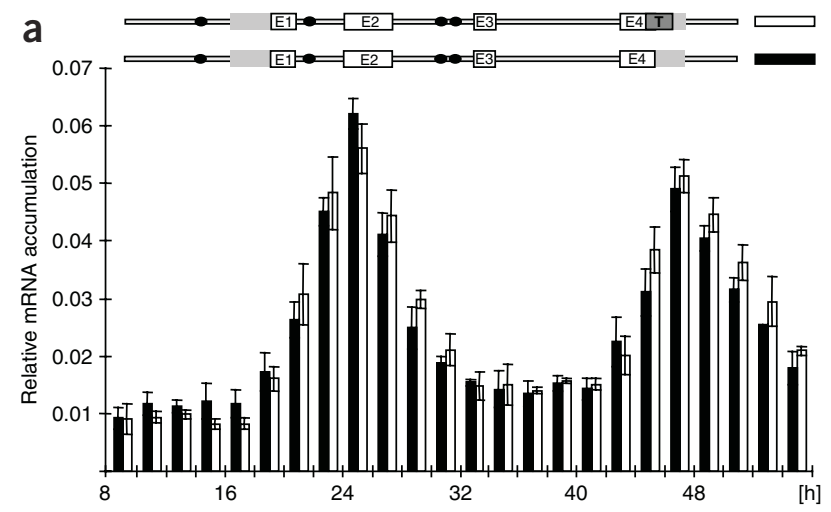

C
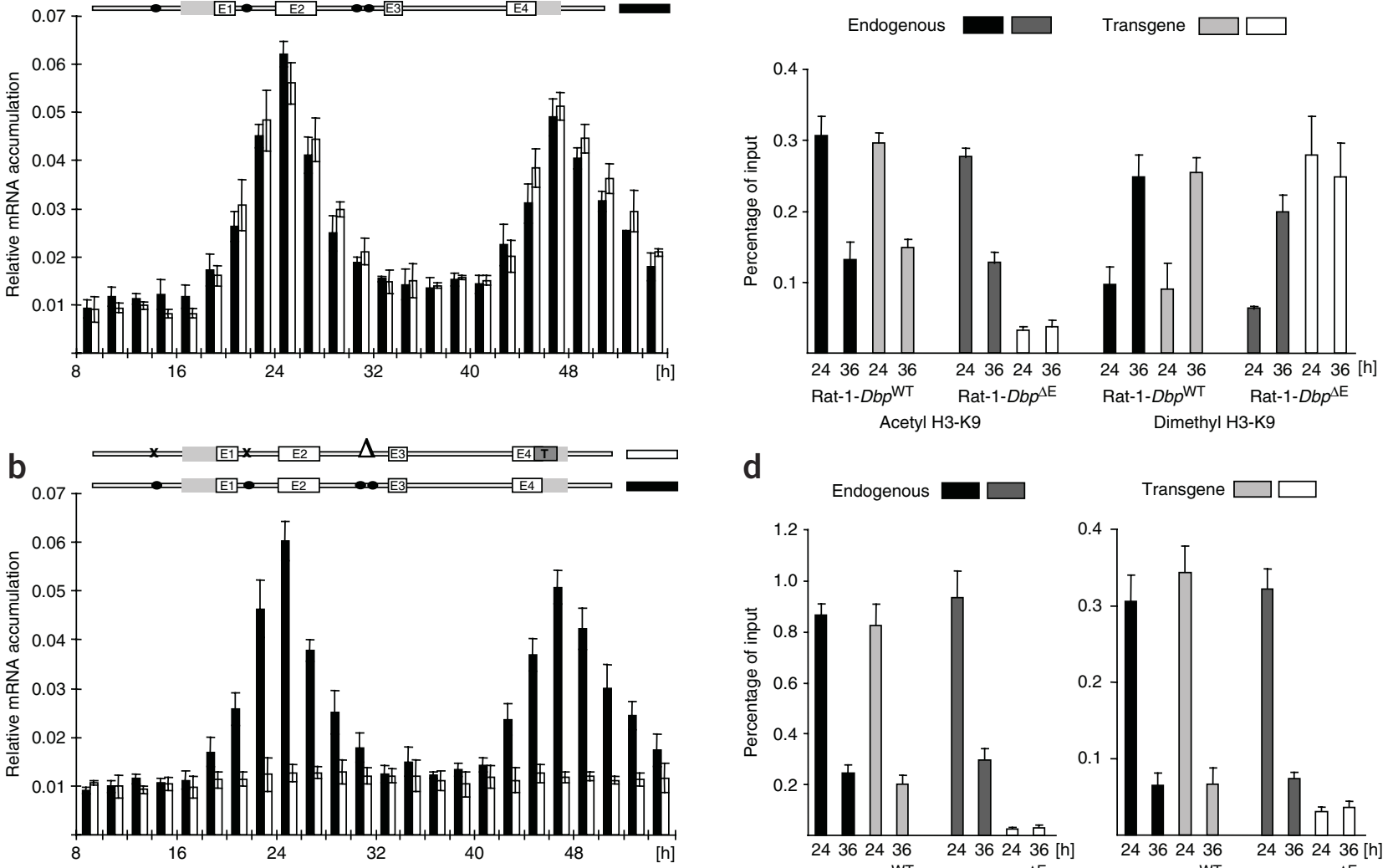

d
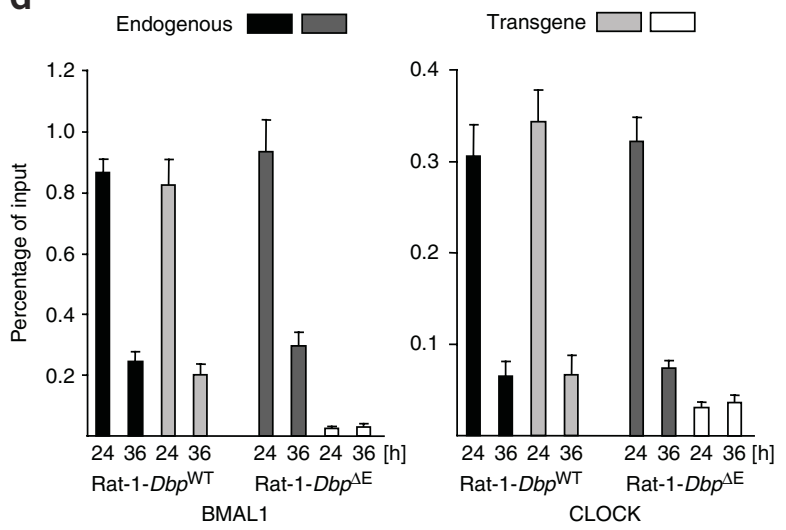

Figure 4 E-box motifs are required for circadian Dbp transcription and histone H3 modifications. (a) Dbp mRNA accumulation in Rat- ${ }^{\text {WT }}$ cells. Total RNA was extracted at the indicated time points after induction with dexamethasone ${ }^{30}$, and the accumulation of the endogenous Dbp mRNA (filled bars) or of the $D b p$ transgene (open bars) was determined by real-time RT-PCR and normalized to Gapdh. The values obtained for endogenous $D b p$ mRNA were divided by two, to allow for a direct comparison with the accumulation of transgene-encoded mRNA (note that Rat-1 ${ }^{\text {WT }}$ cells are haploid for the transgene). Shown are the averages of four pairwise comparisons ( \pm s.d.). (b) mRNA accumulation in Rat- $1^{\Delta \mathrm{E}}$ cells. Data are presented as in a. (c) ChIP analysis for H3 modifications. The presence of acetylated or dimethylated H3-K9 at the endogenous Dbp allele or transgenic Dbp allele was detected in the two different cell lines $24 \mathrm{~h}$ or $36 \mathrm{~h}$ after induction with dexamethasone, using two different probes to discriminate between rat and mouse intron 1 . (d) ChIP analysis for BMAL1 and CLOCK. E1 to E4: exons 1 to 4 . T refers to the tag inserted into the Dbp transgene.

day (Fig. 4b). H3-K9 acetylation and dimethylation remained at minimal and maximal levels, respectively, over this transgene (Fig. 4c). In addition, binding of BMAL1 and CLOCK was markedly reduced in Rat-1-Dbp ${ }^{\Delta \mathrm{E}}$ cells (Fig. 4d). Taken together, these observations indicate that E-box motifs, and consequently the transcriptional activators BMAL1 and CLOCK, are required for both circadian transcription and chromatin modifications.

In conclusion, we present evidence that circadian $D b p$ transcription is accompanied by marked, but reversible, changes in chromatin structure, and that the rhythmic binding of BMAL1 and CLOCK seems to be a prerequisite for these dynamic changes in chromatin modulation. As illustrated by the schematic in Figure 5, part of the Dbp locus may change from a euchromatin-like to a heterochromatinlike state every day. The chromatin compaction may allow a very efficient silencing of the gene, perhaps even in the absence of chromatin-bound repressors (such as CRY and PER). Because fewer than $0.1 \%$ of cells are replicating their DNA in the liver of adult mice at a given time point ${ }^{22}$, the circadian changes in histone methylation within $D b p$ chromatin must occur in the absence of DNA replication. We can think of at least two scenarios that would be compatible with this finding. First, as yet unknown histone demethylases might remove the H3-K9 dimethyl and H3-K4 trimethyl groups every day from nucleosomes that remain associated with the $D b p$ locus for extended time periods. Recently, lysine-specific demethylase 1 (LSD1) has been demonstrated to remove dimethyl groups from Lys4 or Lys9 of histone H3 (refs. 23-25). Second, histones or entire nucleosomes might be exchanged every day, either by de novo synthesized histones or by histones and nucleosomes previously associated with other chromatin regions ${ }^{26,27}$. The second scenario is favored by our observation that histone density undergoes roughly twofold circadian oscillation within the $D b p$ locus. Our demonstration that circadian transcription and the associated changes in chromatin structure can be investigated in synchronized tissue culture cells should greatly facilitate future studies addressing the molecular mechanisms responsible for circadian chromatin transitions.

\section{METHODS}

Animals. All animal care and handling was performed according to the State of Geneva's law for animal protection authorized by the Office Veterinaire Cantonal de Genève. Three- to six-month-old C57BL/6 mice and Per1 ${ }^{-1-}$ Per2 ${ }^{\mathrm{Brdm} / \mathrm{Brdm}}$ mice $^{28}$ (a gift of U. Albrecht, Fribourg, Switzerland) were housed under a 12-h light/12-h dark regimen. 


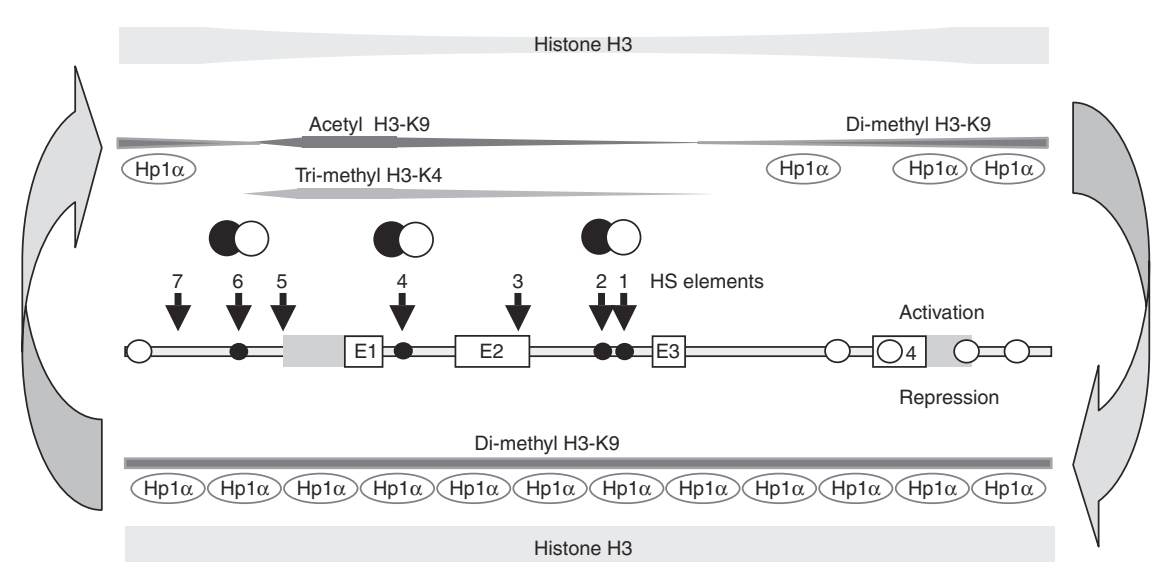

Figure 5 Schematic illustrating the dynamic chromatin changes accompanying circadian $D b p$ transcription. Circadian changes between activation and repression coincide with the presence of markers for active transcription (acetylated $\mathrm{H} 3-\mathrm{K} 9$ and trimethylated $\mathrm{H} 3-\mathrm{K} 4$ ) and the spreading of markers diagnostic for heterochromatin (dimethylated $\mathrm{H} 3-\mathrm{K} 9$ and $\mathrm{Hp} 1 \alpha$ ), respectively. The schematic $D b p$ gene structure shown in the center is the same as that shown in Figure 1. Small open circles on the gene represent sites of CpG methylation (Supplementary Fig. 2 and Supplementary Methods online), and filled and open circles paired together indicate the circadian binding of BMAL1 and CLOCK heterodimers to E-box motifs (small filled circles on the gene). We determined the relative density only of histone $\mathrm{H} 3$ (indicated by width of gray bars at top and bottom), but we assume that the density of the remaining core histones undergoes similar circadian changes.

Chromatin immunoprecipitations. Livers from mice were immediately homogenized in $4 \mathrm{ml}$ per liver of $1 \times$ PBS including $1 \%$ formaldehyde, and the homogenate was kept for $5 \mathrm{~min}$ at $25^{\circ} \mathrm{C}$. Cross-linking reactions were stopped by the addition of $25 \mathrm{ml}$ of ice-cold $2.2 \mathrm{M}$ sucrose in $150 \mathrm{mM}$ glycine, $10 \mathrm{mM}$ HEPES $\mathrm{pH} 7.6,15 \mathrm{mM} \mathrm{KCl}, 2 \mathrm{mM}$ EDTA, $0.15 \mathrm{mM}$ spermine, $0.5 \mathrm{mM}$ spermidine, $0.5 \mathrm{mM}$ DTT and $0.5 \mathrm{mM}$ PMSF. The homogenate was layered on top of a $10 \mathrm{ml}$ cushion of $2.05 \mathrm{M}$ sucrose (containing the same ingredients and including $10 \%$ glycerol and $125 \mathrm{mM}$ glycine) and centrifuged for $30 \mathrm{~min}$ at $24,000 \mathrm{rpm}(100,000 \mathrm{~g})$ at $1{ }^{\circ} \mathrm{C}$ in a Beckmann SW28 rotor. The nuclei were resuspended in $1 \mathrm{ml}$ of $20 \mathrm{mM}$ Tris, $\mathrm{pH} 7.5,150 \mathrm{mM} \mathrm{NaCl}, 2 \mathrm{mM}$ EDTA, transferred to a $4-\mathrm{ml}$ centrifuge tube and sedimented at $1,500 \mathrm{~g}$ in a Heraeus Labofuge 400 for $1 \mathrm{~min}$. They were then resuspended in $1.5 \mathrm{ml}$ per liver of the same buffer supplemented with SDS (final concentration, 1\%), and sonicated for $15 \mathrm{~s}$ on ice ten times. The fragmented cross-linked chromatin was then diluted tenfold with the same buffer containing $1 \%$ Triton X-100.

About $2 \times 10^{6}$ genomic copies were used as starting material for DNA quantification experiments. Coimmunoprecipitated DNA fragments captured by protein A-agarose beads were washed according to ref. 29 , and the crosslinks were reversed overnight at $65{ }^{\circ} \mathrm{C}$ in $100 \mu \mathrm{l}$ of $20 \mathrm{mM}$ Tris $\mathrm{pH} 7.5,0.1 \%$ NP-40, $10 \mu \mathrm{g} \mathrm{ml}^{-1}$ BSA, $0.1 \mathrm{mM}$ EDTA, $0.02 \mu \mathrm{g} \mu^{-1}$ salmon sperm DNA. Four microliters of each reaction were directly used in $20-\mu \mathrm{l}$ real-time PCR reactions using an ABI 7700 machine (Applied Biosystems). The amount of DNA was quantified against a $2^{-n}$ calibration curve prepared from mouse genomic DNA. Experiments were performed with at least two independent preparations of mouse liver chromatin. The primers and Taqman probes used in these experiments are listed in Supplementary Table 1 online.

Antibodies were purchased from Abcam (acetylated H3-K9, trimethylated $\mathrm{H} 3-\mathrm{K} 4$, or Hpl $\alpha$ ) or Upstate (dimethylated H3-K9). The specificity of these antibodies was verified by competition experiments with peptides obtained from the same sources; experiments with the antibody against trimethylated H3-K4 were performed in the presence of dimethylated H3-K4 peptide to reduce cross-reactivity against this modification (about 10\%). Antibodies directed against BMAL1 or CLOCK have been described ${ }^{6}$.

Protein blot and electrophoretic mobility shift assays. The preparation of proteins from mouse liver nuclei and analysis by protein blotting on 5\% SDSPAGE was performed as described previously ${ }^{6}$. Protein-DNA complexes were obtained and resolved as described previously ${ }^{6}$. The high-affinity binding site used in these experiments (Supplementary Table 1) allowed the detection of BMAL1 and CLOCK heterodimers by conventional one-dimensional EMSA, in contrast to a previously used intronic site whose complexes with CLOCK and BMAL1 could be visualized only by a two-dimensional EMSA technique $^{6}$. Supershift-EMSA experiments were performed by adding $0.5 \mu \mathrm{l}$ of the indicated antibodies to the binding reactions before gel electrophoresis. Two gel types and electrophoresis conditions were used in EMSA experiments. In the 'non-stringent method', protein-DNA complexes were separated on composite $3.5 \%$ polyacrylamide $/ 0.5 \%$ agarose $/ 0.4 \times$ TBE native gels at $4{ }^{\circ} \mathrm{C}$. In the 'stringent method', complexes were resolved on $2.5 \%$ polyacrylamide/ $0.75 \%$ agarose $/ 0.25 \times$ TBE native gels at $25{ }^{\circ} \mathrm{C}$.

Rat-1 cell lines with tagged $D b p$ reporter genes. A Rat-1 cell line carrying a single FRT site was generated and tested according to the manufacturer's instructions (Invitrogen). Tagged alleles were obtained by inserting a TetR fragment into the EcoRV site of a 7-kb BglII-fragment of the mouse $D b p$ gene, which was cloned into pcDNA5/FRT (Invitrogen). The promoter-proximal E-box motif (position $166 \pm 3 \mathrm{bp}, \mathrm{U} 29762$ ) and the intron $1 \mathrm{E}$ box motif (position $1438 \pm 3 \mathrm{bp}$ ) were replaced by BglII restriction sites using site-directed mutagenesis, and the intron 2 E-box motifs (2978 to 3091, U29762) were deleted by digestion with PmlI and religation in the presence of a HindIII-linker. Stable rat-1 cell lines were generated by site-specific recombination using FLP recombinase and tested according to the manufacturer's instructions. After synchronization of circadian gene expression by a dexamethasone shock ${ }^{30}$, total RNA was isolated with a RNA II kit (Machery-Nagel). RNA accumulation was monitored by multiplex real-time reverse transcription (RT)-PCR and was normalized to glyceraldehyde-3-phosphate dehydrogenase (Gapdh) mRNA accumulation. ChIP experiments on these cell lines were performed according to ref. 29 except that the cross-links were reversed as described above.

Note: Supplementary information is available on the Nature Genetics website.

\section{ACKNOWLEDGMENTS}

We are grateful to U. Albrecht for supplying us with $\operatorname{Per}^{-/-} / \operatorname{Per} 2^{\mathrm{Brdm} / \mathrm{Brdm}}$ mice; D. Shore and R. Sternglanz for discussions, S. Brown, H. Reinke, D. Gatfield and the other members of our lab for discussions and N. Roggli for preparation of the figures. This work was supported by the Canton of Geneva, the Swiss National Science Foundation (through an individual grant to U.S. and the Swiss National Center for Competence in Research program grant 'Frontiers in Genetics'), the Louis Jeantet Foundation of Medicine and the Bonizzi-Theler Stiftung.

\section{COMPETING INTERESTS STATEMENT}

The authors declare that they have no competing financial interests.

1. Reppert, S.M. \& Weaver, D.R. Coordination of circadian timing in mammals. Nature 418, 935-941 (2002).

2. Albrecht, U. The mammalian circadian clock: a network of gene expression. Front. Biosci. 9, 48-55 (2004).

3. Lowrey, P.L. \& Takahashi, J.S. Genetics of the mammalian circadian system: Photic entrainment, circadian pacemaker mechanisms, and posttranslational regulation. Annu. Rev. Genet. 34, 533-562 (2000).

4. Wuarin, J. \& Schibler, U. Expression of the liver-enriched transcriptional activator protein DBP follows a stringent circadian rhythm. Cell 63, 1257-1266 (1990).

5. Lopez-Molina, L., Conquet, F., Dubois-Dauphin, M. \& Schibler, U. The Dbp gene is expressed according to a circadian rhythm in the suprachiasmatic nucleus and influences circadian behavior. EMBO J. 16, 6762-6771 (1997). 
6. Ripperger, J.A., Shearman, L.P., Reppert, S.M. \& Schibler, U. CLOCK, an essential pacemaker component, controls expression of the circadian transcription factor DBP. Genes Dev. 14, 679-689 (2000).

7. Bunger, M.K. et al. Mop3 is an essential component of the master circadian pacemaker in mammals. Cell 103, 1009-1017 (2000).

8. Lee, C., Etchegaray, J.P., Cagampang, F.R., Loudon, A.S. \& Reppert, S.M. Posttranslational mechanisms regulate the mammalian circadian clock. Cell 107, 855-867 (2001).

9. Etchegaray, J.P., Lee, C., Wade, P.A. \& Reppert, S.M. Rhythmic histone acetylation underlies transcription in the mammalian circadian clock. Nature 421, 177-182 (2003).

10. Schafmeier, T. et al. Transcriptional feedback of Neurospora circadian clock gene by phosphorylation-dependent inactivation of its transcription factor. Cell 122, 235-246 (2005).

11. Fischle, W., Wang, Y. \& Allis, C.D. Histone and chromatin cross-talk. Curr. Opin. Cell Biol. 15, 172-183 (2003).

12. Margueron, R., Trojer, P. \& Reinberg, D. The key to development: interpreting the histone code? Curr. Opin. Genet. Dev. 15, 163-176 (2005).

13. Ng, H.H., Robert, F., Young, R.A. \& Struhl, K. Targeted recruitment of Set1 histone methylase by elongating Pol II provides a localized mark and memory of recent transcriptional activity. Mol. Cell 11, 709-719 (2003).

14. Liang, G. et al. Distinct localization of histone H3 acetylation and H3-K4 methylation to the transcription start sites in the human genome. Proc. Natl. Acad. Sci. USA 101, 7357-7362 (2004).

15. Bannister, A.J. et al. Selective recognition of methylated lysine 9 on histone $\mathrm{H} 3$ by the HP1 chromo domain. Nature 410, 120-124 (2001).

16. Lachner, M., O'Carroll, D., Rea, S., Mechtler, K. \& Jenuwein, T. Methylation of histone H3 lysine 9 creates a binding site for HP1 proteins. Nature 410, 116-120 (2001).
17. Sarraf, S.A. \& Stancheva, I. Methyl-CpG binding protein MBD1 couples histone H3 methylation at lysine 9 by SETDB1 to DNA replication and chromatin assembly. Mol. Cell 15, 595-605 (2004).

18. Balsalobre, A., Damiola, F. \& Schibler, U. A serum shock induces circadian gene expression in mammalian tissue culture cells. Cell 93, 929-937 (1998).

19. Akashi, M. \& Nishida, E. Involvement of the MAP kinase cascade in resetting of the mammalian circadian clock. Genes Dev. 14, 645-649 (2000).

20. Nagoshi, E. et al. Circadian gene expression in individual fibroblasts; cell-autonomous and self-sustained oscillators pass time to daughter cells. Cell 119, 693-705 (2004).

21. Wirth, D. \& Hauser, H. Flp-mediated integration of expression cassettes into FRT-tagged chromosomal loci in mammalian cells. Methods Mol. Biol. 267, 467-476 (2004).

22. Yin, L. et al. Control of mouse hepatocyte proliferation and ploidy by p53 and p53ser246 mutation in vivo. Hepatology 27, 73-80 (1998).

23. Shi, Y. et al. Histone demethylation mediated by the nuclear amine oxidase homolog LSD1. Cell 119, 941-953 (2004).

24. Lee, M.G., Wynder, C., Cooch, N. \& Shiekhattar, R. An essential role for CoREST in nucleosomal histone 3 lysine 4 demethylation. Nature 437, 432-435 (2005).

25. Metzger, E. et al. LSD1 demethylates repressive histone marks to promote androgenreceptor-dependent transcription. Nature 437, 436-439 (2005).

26. Kimura, $\mathrm{H}$. Histone dynamics in living cells revealed by photobleaching. DNA Repair (Amst.) 4, 939-950 (2005).

27. Kimura, H. \& Cook, P.R. Kinetics of core histones in living human cells: little exchange of $\mathrm{H} 3$ and $\mathrm{H} 4$ and some rapid exchange of H2B. J. Cell Biol. 153, 1341-1353 (2001).

28. Zheng, B. et al. Nonredundant roles of the mPer1 and mPer2 genes in the mammalian circadian clock. Cell 105, 683-694 (2001).

29. Shang, Y., Hu, X., DiRenzo, J., Lazar, M.A. \& Brown, M. Cofactor dynamics and sufficiency in estrogen receptor-regulated transcription. Cell 103, 843-852 (2000).

30. Balsalobre, A. et al. Resetting of circadian time in peripheral tissues by glucocorticoid signaling. Science 289, 2344-2347 (2000). 